\title{
Ambiente familiar e consumo de álcool e tabaco entre adolescentes
}

\author{
Familiar environment and use of alcohol and tobacco among teenagers
}

\section{Rafael Souza Moreno ${ }^{1}$, Renato Nabas Ventura², José Roberto S. Brêtas ${ }^{3}$}

\section{RESUMO}

Objetivo: Analisar a influência do ambiente familiar em relação ao uso de álcool e tabaco pelos adolescentes.

Métodos: Trata-se de estudo descritivo, elaborado a partir da análise e aprofundamento da categoria referente ao uso de drogas lícitas e a influência familiar, presente em um questionário semiestruturado, contendo ao todo 25 questões sobre o uso álcool e tabaco, realizado junto a 1.533 adolescentes de ambos os sexos, tendo por critérios de inclusão: adolescentes entre dez e 20 anos de idade, matriculados e frequentando regularmente a sexta, a sétima ou a oitava séries do ensino fundamental e o primeiro, o segundo ou o terceiro anos do ensino médio das escolas estaduais situadas nas regiões de Santo Eduardo e Santa Emília, no município de Embu, no período matutino, e que aceitaram participar das oficinas de prevenção e promoção da saúde realizadas pelo Projeto Corporalidade e Promoção da Saúde. A análise estatística foi aplicada por meio do teste do qui-quadrado, ao nível de significância $p<0,05$, tendo como medida de risco a aplicação do Odds Ratio, com intervalo de confiança de $95 \%$.

Resultados: Os dados demonstraram que $66 \%$ dos adolescentes que não experimentaram bebidas alcoólicas não possuem familiares que bebem frequentemente $(p<0,001)$ e $84 \%$ dos que são fumantes apresentam familiares que fumam $(p<0,001)$.

Conclusões: $\mathrm{O}$ ambiente familiar induz e facilita o uso de álcool e tabaco por adolescentes, tornando-se fundamental a utilização deste conhecimento na elaboração de projetos de prevenção e educação em saúde.

Palavras-chave: adolescente; consumo de bebidas alcoólicas; tabagismo; relações familiares.

\section{ABSTRACT}

Objective: To analyze the influence of the family environment in relation to alcohol and tobacco use among adolescents.

Methods: This was a descriptive study consisting of in-depth analysis on the topic of family influence related to legal drug use. A semi-structured questionnaire containing 25 questions on alcohol and tobacco use was completed by 1,533 adolescents of both genders. Inclusion criteria were: adolescents aged ten to 20 years who were regularly attending morning classes in the sixth, seventh or eighth years of elementary education or in the first, second or third years of high school education at state schools in two regions of the municipality of Embu, São Paulo, Brazil. Subjects agreed to participate in preventive health promotion workshops of the Corporality and Health Promotion Project. Statistical analysis applied odds ratio, with a $95 \%$ confidence interval and chi-square test, being significant $p<0.05$.

Results: $66 \%$ of the adolescents who had not tried alcoholic drinks did not have any family members who were frequent drinkers $(p<0.001)$ and $84 \%$ of those who smoked had family members who were smokers $(p<0.001)$.

Conclusions: The family environment induces and facilitates alcohol and tobacco use. It is extremely important to use this knowledge to design preventive health education projects.

Key-words: adolescent; alcohol drinking; smoking; family relations.
Instituição: Universidade Federal de São Paulo (Unifesp), São Paulo, SP, Brasil

${ }^{1}$ Enfermeiro do Hospital São Paulo; Especialista em Enfermagem em Nefrologia; Membro do Grupo de Estudos Sobre Corporalidade e Promoção da Saúde (Gecopros), São Paulo, SP, Brasil

${ }^{2}$ Médico do Departamento de Pediatria da Unifesp, São Paulo, SP, Brasil ${ }^{3}$ Psicólogo; Professor adjunto do Departamento de Enfermagem da Unifesp; Líder do Gecopros, São Paulo, SP, Brasil
Endereço para correspondência:

Rafael Souza Moreno

Rua José Moreno Mostazo, 700

CEP 07500-000 - Santa Isabel/SP

E-mail: splintermoreno@yahoo.com.br

Fonte financiadora: Fundação de Amparo a Pesquisa do Estado de São Paulo (Fapesp), processo 06/56390-8. 


\section{Introdução}

Historicamente, a utilização de substâncias naturais e produtos para obter alterações psicossomáticas, com o intuito de conferir ao usuário poderes de ver, sentir ou pressentir o que outros na sobriedade não conseguem, confunde-se com a evolução natural da humanidade ${ }^{(1)}$.

Em relação ao consumo de tabaco pela população mundial, estima-se haver atualmente no mundo aproximadamente um bilhão e 200 milhões de fumantes ativos, dos quais 960 milhões são dependentes da nicotina ${ }^{(2)}$. A ingestão de álcool é ainda mais alarmante: mais de $80 \%$ da população mundial adulta utiliza ou já experimentou algum tipo de bebida alcoólica, sendo que apenas cerca de $20 \%$ dela pode ser considerada completamente abstêmia ${ }^{(3)}$.

O público adolescente representa a parcela populacional mais vulnerável para se envolver com drogas lícitas, das quais se destaca, pela significativa prevalência, o álcool, que atualmente possui a faixa etária de experimentação mais jovem, em torno de 12,5 anos; em seguida, encontra-se o tabaco $(12,8 \text { anos })^{(4)}$. Sabe-se que, quanto mais precoce o contato com o álcool e o tabaco, maiores serão os riscos futuros de agravos à saúde ${ }^{(5,6)}$.

$\mathrm{O}$ adolescente vivencia um período de intensa transformação biopsicossocial, no qual o que importa é a busca por uma nova identidade e independência individual, absorvendo atitudes, ações e costumes do meio em que está inserido. Neste ponto, a família se constitui socialmente em uma unidade primordial no âmbito da construção, formação e desenvolvimento dos indivíduos que a compõem, transmitindo às gerações valores, regras, costumes, ideais, além de modelos e padrões de comportamento ${ }^{(7,8)}$.

Ao debater a questão do início precoce do uso e consumo de drogas lícitas, diversos autores apontam como influências determinantes da experimentação a pressão dos amigos e o ambiente familiar ${ }^{(9,10)}$. Segundo Minayo, ao abordar o domínio familiar e a sua influência na utilização de substâncias psicoativas pelos adolescentes, destacam-se como fatores de proteção contra o uso de drogas o estabelecimento de fortes vínculos entre pais e filhos, a criação de regras e a imposição de limites claros e coerentes, além da monitorização, supervisão e apoio aos jovens nas suas decisões e atitudes, adotando principalmente o diálogo como prática comum na rotina familiar ${ }^{(11)}$. $\mathrm{O}$ ambiente familiar acaba por influenciar o jovem a experimentar as drogas utilizadas pelos pais e parentes próximos ${ }^{(11,12)}$, característica esta que preocupa, uma vez que o uso cotidiano de álcool e tabaco em domicílios brasileiro é elevado ${ }^{(13,14)}$.

Durante o primeiro levantamento domiciliar realizado pelo Centro Brasileiro de Informações Sobre Drogas Psicotrópicas (Cebrid) em 2001, nas 107 cidades brasileiras com população superior a 200 mil habitantes na faixa etária compreendida entre 12 e 65 anos, constatou-se que, dos 8.589 entrevistados, o uso cotidiano do tabaco foi relatado por $41 \%$, enquanto que o de bebidas alcoólicas por 69\% ${ }^{(13)}$. Já no levantamento domiciliar seguinte, em 2005, realizado com 7.939 indivíduos nas 108 maiores cidades brasileiras, tomando a mesma faixa etária, observou-se que o uso cotidiano de álcool aumentou para $75 \%$ e o de tabaco para $44 \%{ }^{(14)}$.

Neste contexto, o presente estudo teve como objetivo estudar a influência do ambiente familiar no uso de drogas lícitas (álcool e tabaco) por adolescentes das escolas municipais das regiões do Jardim Santo Eduardo e Santa Emília, município de Embu, buscando - por meio dos resultados obtidos - propor ações referentes à prevenção e à educação em saúde dos estudantes destas escolas, com a criação de material didático relativo aos riscos e consequências do uso das drogas lícitas, dentro da perspectiva do projeto de extensão universitária denominado Corporalidade e Promoção da Saúde.

\section{Métodos}

Trata-se de um estudo descritivo, elaborado a partir da análise e aprofundamento das discussões referentes ao uso de drogas lícitas e a influência familiar. Aplicou-se um questionário semiestruturado com questões fechadas, sendo realizado primeiramente um pré-teste com 48 alunos. Após as correções, o teste foi distribuído em sala de aula sob a supervisão dos pesquisadores ao final do segundo semestre de 2006 e início do primeiro semestre de 2007, com 25 questões semiestruturadas sobre o uso de álcool e tabaco, parte do Projeto 'O uso de drogas lícitas na visão de adolescentes que frequentam escolas do município de Embu, SP'. O projeto foi aprovado pelo Comitê de Ética da Unifesp e financiado pela Fundação de Amparo à Pesquisa do Estado de São Paulo (Fapesp).

O questionário foi aplicado a 1.541 adolescentes que obedeceram aos seguintes critérios de inclusão: faixa etária entre dez e 20 anos de idade, matriculados e frequentando regularmente a sexta, sétima ou oitava séries de ensino fundamental e o primeiro, segundo ou terceiro anos do ensino médio das escolas estaduais situadas nas regiões de 
Santo Eduardo e Santa Emília, no município de Embu, no período matutino e que aceitaram participar das oficinas de educação em saúde realizadas pelo Projeto Corporalidade e Promoção da Saúde, no contexto do Programa de Integração Docente-Assistencial do Embu (Pida-Embu) da Unifesp.

Em relação às variáveis do estudo, adotou-se como critério de definição: fez uso na vida de bebida alcoólica ${ }^{(4)}$ - quando a pessoa fez uso pelo uma vez na vida; uso frequente de bebidas alcoólicas ${ }^{(4)}$ - quando a pessoa ingeriu seis ou mais doses de qualquer bebida nos 30 dias que antecederam a pesquisa; ser fumante ${ }^{(15)}$ - ter fumado um ou mais cigarros nos últimos 30 dias.

Para análise estatística, aplicou-se o teste do qui-quadrado e, nos casos em que este não se mostrasse adequado, foi utilizado o teste da razão de verossimilhança, com um nível de significância de 5\%, realizadas por meio do SPSS 12.0 for Windows, além da aplicação do Odds Ratio e intervalo de confiança de $95 \%$, calculados pelo método de Woolf ${ }^{(16)}$.

\section{Resultados}

Dos 1.541 adolescentes que obedeceram aos critérios de inclusão no estudo, foram excluídos oito questionários devido ao preenchimento incorreto dos dados.

A população retratada nesta pesquisa foi composta por 1.533 adolescentes, sendo $799(52 \%)$ do sexo masculino. A

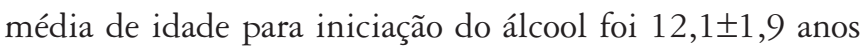
(variação: 3-20) e para o tabaco 12,6 $\pm 1,5$ anos (variação: 9-16). A maioria dos alunos (78\%) se encontrava na faixa etária entre os 12 e 16 anos. Em relação ao estado civil, 98\% eram solteiros. Quanto à escolaridade, a maior parcela dos estudantes (42\%) estava matriculada e frequentava regularmente a oitava serie do ensino fundamental.

Os dados da Tabela 1 se referem aos adolescentes com familiares que bebiam frequentemente, observando-se uma associação significante entre a variável adolescente já ter experimentado bebida alcoólica ou fazer uso na vida e possuir familiar que bebe frequentemente $(p<0,001)$.

Ao analisar a questão da influência do ambiente doméstico e o uso de drogas lícitas, no caso específico do consumo de bebidas alcoólicas, 206 (43\%) adolescentes apontaram a figura paterna como principal familiar que bebe em seus lares. Dentre os demais citados, os tios ocupam o segundo lugar com $15 \%$, seguidos pelos irmãos (9\%) e mãe, com $6 \%$, não havendo diferença significante entre meninos e meninas em relação a quanto cada familiar influencia o uso de álcool, como pode ser observado na Tabela 2 .

Dentre os adolescentes fumantes estratificados pelo comportamento tabagístico dos seus familiares, houve associação estatística entre possuir no ambiente doméstico familiares fumantes e o fato de o adolescente também apresentar esse hábito (Tabela 3). Os familiares mais citados foram o pai e a mãe, correspondendo a 35 do total dos adolescentes (56\%), dos quais 16 (26\%) destacaram o pai e 19 (31\%), a mãe. Merece atenção a prevalência elevada de tios $(6,5 \%)$ e irmãos $(9,7 \%)$ fumantes (Tabela 4).

\section{Discussão}

Os dados expostos nesta pesquisa corresponderam a adolescentes residentes em uma região bastante afetada pelos altos índices de desemprego, violência e exclusão social, refletidos nas elevadas taxas de mortalidade por causas externas, sobretudo homicídios, envolvendo adolescentes e adultos jovens (214 por 100.000 habitantes $)^{(17,18)}$.

O município de Embu compõe a sub-região Sudoeste da região metropolitana de São Paulo, caracterizada por pequena concentração industrial, baixo potencial de crescimento econômico e amplas áreas de proteção de mananciais. $\mathrm{O}$ município é uma estância turística, $100 \%$ urbanizada, com uma população estimada em 244.642 habitantes. A população de faixa etária entre zero e 19 anos perfaz $38 \%$ do total. A

Tabela 1 - Frequência absoluta e relativa de adolescentes que experimentaram bebidas alcoólicas, segundo os hábitos de seus familiares

\begin{tabular}{|c|c|c|c|c|c|c|c|c|}
\hline & \multicolumn{6}{|c|}{ Experimentou bebida alcoólica } & \multirow{3}{*}{ Valor $p$} & \multirow{3}{*}{ OR (IC95\%) } \\
\hline & \multicolumn{2}{|c|}{ Sim } & \multicolumn{2}{|c|}{ Não } & \multicolumn{2}{|c|}{ Total } & & \\
\hline & $\mathbf{n}$ & $\%$ & $\mathbf{n}$ & $\%$ & $\mathbf{n}$ & $\%$ & & \\
\hline \multicolumn{9}{|c|}{ Familiar que bebe frequentemente } \\
\hline Sim & 479 & 53,6 & 220 & 34,4 & 699 & 45,6 & $<0,001$ & $2,2(2,65-1,79)$ \\
\hline Não & 415 & 46,4 & 419 & 65,6 & 834 & 54,4 & & \\
\hline Total & 894 & & 639 & & 1533 & & & \\
\hline
\end{tabular}


Tabela 2 - Distribuição dos familiares que bebem frequentemente, citados pelos adolescentes que fizeram uso na vida de bebidas alcoólicas, segundo o sexo

\begin{tabular}{|c|c|c|c|c|c|c|c|}
\hline & \multicolumn{6}{|c|}{ Adolescentes que fizeram uso na vida } & \multirow{3}{*}{ Valor $p$} \\
\hline & \multicolumn{2}{|c|}{ Masculino } & \multicolumn{2}{|c|}{ Feminino } & \multicolumn{2}{|c|}{ Total } & \\
\hline & $\mathbf{n}$ & $\%$ & $\mathbf{n}$ & $\%$ & $\mathbf{n}$ & $\%$ & \\
\hline Familiar que bebe frequentemente & & & & & & & NS \\
\hline Pai & 100 & 41,0 & 106 & 45,1 & 206 & 43,0 & NS \\
\hline Mãe & 16 & 6,6 & 14 & 6,1 & 30 & 6,3 & NS \\
\hline Irmãos & 21 & 8,6 & 23 & 9,8 & 44 & 9,2 & NS \\
\hline Avós & 6 & 2,5 & 5 & 2,1 & 11 & 2,3 & NS \\
\hline Tios & 39 & 16,0 & 39 & 14,5 & 73 & 15,2 & NS \\
\hline Primos & 2 & 0,8 & 1 & 0,4 & 3 & 0,6 & NS \\
\hline Pai/mãe & 10 & 4,1 & 12 & 5,1 & 22 & 4,6 & NS \\
\hline Outros & 10 & 4,1 & 15 & 6,4 & 25 & 5,2 & NS \\
\hline Não especificou & 40 & 16,3 & 25 & 10,6 & 65 & 13,6 & NS \\
\hline
\end{tabular}

NS: não significante.

Tabela 3 - Frequência absoluta e relativa de adolescentes fumantes, segundo os hábitos tabagísticos de seus familiares

\begin{tabular}{|c|c|c|c|c|c|c|c|c|}
\hline \multirow{3}{*}{ Variáveis } & \multicolumn{6}{|c|}{ Adolescente fumante } & \multirow{3}{*}{ Valor $p$} & \multirow{3}{*}{ OR (IC95\%) } \\
\hline & \multicolumn{2}{|c|}{ Sim } & \multicolumn{2}{|c|}{ Não } & \multicolumn{2}{|c|}{ Total } & & \\
\hline & $\mathbf{n}$ & $\%$ & $\mathbf{n}$ & $\%$ & $\mathbf{n}$ & $\%$ & & \\
\hline Familiar fumante & & & & & & & $<0,001$ & $3,9(2,08-7,29)$ \\
\hline Sim & 62 & 83,8 & 835 & 57,2 & 897 & 58,5 & & \\
\hline Não & 12 & 16,2 & 624 & 42,8 & 636 & 41,5 & & \\
\hline Total & 74 & & 1459 & & 1533 & & & \\
\hline
\end{tabular}

Tabela 4 - Distribuição dos familiares que fumam, citados pelos adolescentes que apresentam o hábito tabagístico, segundo o sexo

\begin{tabular}{|c|c|c|c|c|c|c|c|}
\hline \multirow{3}{*}{ Variáveis } & \multicolumn{6}{|c|}{ Adolescente fumante } & \multirow{3}{*}{ Valor $p$} \\
\hline & \multicolumn{2}{|c|}{ Masculino } & \multicolumn{2}{|c|}{ Feminino } & \multicolumn{2}{|c|}{ Total } & \\
\hline & $\mathrm{n}$ & $\%$ & $\mathrm{n}$ & $\%$ & $\mathrm{n}$ & $\%$ & \\
\hline Familiar fumante & & & & & & & NS \\
\hline Pai & 7 & 26,9 & 9 & 25,0 & 16 & 25,8 & NS \\
\hline Mãe & 9 & 34,7 & 10 & 27,8 & 19 & 30,7 & NS \\
\hline Irmãos & 1 & 3,8 & 5 & 13,9 & 6 & 9,6 & NS \\
\hline Avós & 1 & 3,8 & 0 & - & 1 & 1,6 & NS \\
\hline Tios & 1 & 3,8 & 3 & 8,3 & 4 & 6,5 & NS \\
\hline Pai/mãe & 1 & 3,8 & 1 & 2,8 & 2 & 3,2 & NS \\
\hline Outros & 3 & 11,6 & 4 & 11,1 & 7 & 11,3 & NS \\
\hline Não especificou & 3 & 11,6 & 4 & 11,1 & 7 & 11,3 & NS \\
\hline
\end{tabular}

NS: não significante.

população entre 18 e 24 anos com ensino médio completo representa $29 \%$ e a média dos anos de estudo da população entre 15 e 64 anos é de 6,5 anos $^{(18)}$.

Nesse contexto, a maioria desses jovens vive em um ambiente familiar desfavorecido, com condições socioeconômicas inapropriadas para prover um meio adequado ao desenvolvimento biopsicossocial do indivíduo. Por outro lado, considerando os aspectos próprios da adolescência, observa-se que cada movimento do adolescente em direção à maturidade e ao desenvolvimento produz novas conquistas, mas também novos problemas. Neste sentido, Ana Freud ${ }^{(19)}$ refere que uma mudança em qualquer setor da vida mental perturba o equilíbrio anteriormente estabelecido e impõe a busca por novos compromissos. Diante dessa situação 
de equilíbrio alterado em que se encontra o adolescente, pode ocorrer a ingestão de drogas lícitas e ilícitas, o que se torna cada vez mais comum; dentre as lícitas, pode-se nomear principalmente o álcool e o tabaco. O uso de drogas representa, por vezes, um auxílio para o adolescente superar suas inibições e ousar experimentar situações novas, afirmando-se como igual dentro do seu grupo. Além disso, há a sedução por algo que é proibido e pela curiosidade da experiência.

Estudos mostram que baixo nível de escolaridade, salário não suficiente para satisfazer necessidades básicas, conflitos intra e interfamiliares, falta de disciplina, falta de intimidade dos pais em relação aos filhos, distância dos pais, falta de diálogo entre os membros da família e baixa expectativa do futuro educacional dos filhos são fatores de riscos que levam à indução dos jovens e/ou adolescentes ao consumo de drogas lícitas ${ }^{(7,8,10)}$. Por outro lado, há relatos, na literatura internacional, da associação entre jovens que vivem numa estrutura familiar consolidada com pais unidos e menor propensão ao consumo de drogas lícitas ou ilícitas, ao contrário daqueles em que os pais não vivem juntos ${ }^{(20)}$.

Para Laranjeira e Pinsky ${ }^{(3)}$ a facilitação do uso de bebidas no ambiente doméstico também está associada ao hábito dos pais incentivarem seus filhos a beberem com eles, hábito este que se torna cada vez mais comum nos lares brasileiros. Segundo Kalina ${ }^{(21)}$, a família tem uma participação fundamental, pois quando se defronta com um problema de uso de drogas na adolescência, é necessário reportar ao grupo familiar as suas dificuldades, manifestas ou não. Neste sentido, Outeiral ${ }^{(22)}$ relata que, muitas vezes, observam-se adolescentes em grupos familiares nos quais não há um alcoolista, ou seja, um adicto ao álcool, mas sim uma 'cultura alcoólatra', em que o álcool é idolatrado. Existe em casa um lugar privilegiado - em geral na sala onde estão as garrafas de bebida. Quando chega um amigo ou alguém a quem a família quer prestar uma gentileza, abre-se uma das garrafas e bebe-se algo. O adolescente, vendo esta substância tão venerada, quer usá-la também. Sem falar no fato comum de pais acharem graça e até se sentirem orgulhosos se seu filho se embriaga: "é coisa de homem, acontece...", dizem satisfeitos.

De acordo com Schenker e Minayo ${ }^{(11,23)}$, pais que dialogam frequentemente com seus filhos desde a infância e que conseguem impor limites claros ao longo da sua formação estabelecem uma forte rede de proteção em relação ao consumo de substâncias psicoativas pelos jo- vens durante o período do adolescer. Entretanto, a falta de capacidade dos pais em abordar a questão do uso das drogas ou a falta de discernimento para tratar do assunto com responsabilidade perante os filhos, orientando-os e transmitindo valores a favor de sua saúde, constituem aspectos de não-coibição e favorecem o consumo tanto de álcool como de tabaco ${ }^{(24)}$.

Diante de todos esses acontecimentos transformadores, felizmente a maior parte dos adolescentes consegue a superação, atravessando essa etapa e reencontrando um lugar próprio no mundo, conectado com a vida e projetos pessoais. Para que isso ocorra, os adolescentes necessitam de um meio e de pais, cuja presença estável e coerente proporcione um confronto claro e honesto, estabelecendo os limites necessários. A família, desta maneira, exerce um papel primordial na construção e formação do indivíduo, sendo que problemas de relacionamento entre pais e filhos, ausência de normas e de regras adequadas, uso de drogas pelos pais, além de relações afetivas e emocionais estreitas, tornam-se fatores de risco para o envolvimento com bebidas alcoólicas e tabaco pelos adolescentes.

Segundo pesquisa realizada na Unifesp, o álcool e o tabaco representaram as drogas com a faixa etária de experimentação mais baixa, em torno respectivamente de 12,1 e 13,6 anos. Tais estimativas corroboram nossos achados. Essas médias são preocupantes em termos de saúde pública, uma vez que a iniciação precoce às drogas lícitas potencializa o surgimento de futuros dependentes químicos ${ }^{(4)}$.

A partir dos dados apresentados, observa-se que o ambiente doméstico da população estudada pode estar influenciando os adolescentes quanto ao uso de drogas lícitas (álcool e tabaco). Dados semelhantes em relação ao consumo etílico são notados em pesquisa realizada em 2002 no município de Pelotas (RS), a qual demonstrou que a chance de tabagismo em adolescentes com pai e mãe fumantes é 3,92 vezes maior do que no grupo no qual os pais não são fumantes; entretanto, não houve relação com o consumo de bebidas alcoólicas, discordando da nossa pesquisa nesse ponto ${ }^{(25)}$.

Atualmente, sabe-se que os filhos de dependentes químicos constituem um grupo vulnerável ao desenvolvimento de transtornos psiquiátricos, problemas físico-emocionais, dificuldades escolares, além de possuírem chances elevadas de consumirem substâncias psicoativas, em comparação com os filhos de não dependentes químicos ${ }^{(26)}$.

Em relação aos familiares que utilizam drogas lícitas, citados tanto pelo grupo de adolescentes que ingeriam bebidas alcoólicas como pelo de fumantes, tanto o pai como a 
mãe exercem influência semelhante em meninos e meninas. Torna-se importante ressaltar a presença marcante dos tios e irmãos, representados tanto pelos que fizeram uso de bebidas alcoólicas como pelos fumantes, ou seja, parentes de primeiro grau exercendo influência direta nos jovens. Esses parentes adultos em contato diário com os adolescentes são tratados na literatura como modelos observacionais para os jovens, uma vez que se tornam figuras centrais e necessárias para a fase do adolescer, na qual o indivíduo passa a absorver e a seguir os padrões e costumes de seus familiares ${ }^{(11)}$.

Contudo, o hábito tabagístico não pode ser relacionado apenas a fatores socioambientais, mas também a bases genéticas (fatores intrínsecos). Estudos mostram, atualmente, a expressão de genes relacionados ao tempo de metabolização da nicotina, à sensibilidade ou tolerância à nicotina e ao grau de intensidade da nicotina-dependência. Dessa maneira, existem indivíduos que possuem uma expressão genética que os tornam pouco propensos à ação da nicotina, não sentindo prazer em usá-la, e fumam apenas por estímulo e exposição ao meio em que estão inseridos ${ }^{(2)}$. Assim, o fato de ser fumante ou abstêmio acaba por ser resultado da sensibilidade inicial à nicotina, mas o grau de dependência é determinado pela exposição a essa droga lícita, tendo por influência direta o ambiente familiar e/ou extrafamiliar propensos ao uso do tabaco ${ }^{(2)}$.

\section{Referências bibliográficas}

1. Venâncio J, Jorge MA, Alencar PS, Belmonte PR, Reis VL. Textos de apoio em saúde mental. Rio de Janeiro: Fiocruz; 2003.

2. Rosemberg J. Nicotina: droga universal. São Paulo: SES/CVE; 2003.

3. Laranjeira R, Pinsky I. O alcoolismo. São Paulo: Contexto; 1998.

4. Galduróz JC, Noto AR, Fonseca AM, Carlini EA. V Levantamento nacional sobre o consumo de drogas psicotrópicas entre estudantes do ensino fundamental e médio da rede pública de ensino nas 27 capitais brasileiras, 2004. São Paulo: Cebrid/Departamento de Psicobiologia da Escola Paulista de Medicina; 2004.

5. Cinciripini PM, Hecht SS, Henningfield JE, Manley MW, Kramer BS. Tobacco addiction: implications for treatment and cancer prevention. J Natl Cancer Inst 1997;89:1852-67.

6. Nair BN, Yamaguchi T, Okubo T, Suematsu H, Keizer K, Nakao SI et al. Adolescent alcohol use development and young adult outcomes. Drug Alcohol Depend 1997;49:39-48.

7. Martinez L del C, Ferriani MG. Relação entre as características da adolescente grávida e a resistência contra o consumo de drogas. Rev Latino-Am Enfermagem 2004;12:333-9.

8. Pratta EM, Santos MA. Opiniões dos adolescentes do ensino médio sobre o relacionamento familiar e seus planos para o futuro. Paideia 2007;17: 103-14.

9. Baus J, Kupek E, Pires M. Prevalência e fatores de risco relacionados ao uso de drogas entre escolares. Rev Saude Publica 2002;36:40-6.
Em relação ao álcool, os filhos de pais que fazem o uso indiscriminado apresentam uma propensão quatro vezes maior para o desenvolvimento do alcoolismo do que filhos de pais abstêmios ${ }^{(26)}$.

O estudo, apesar de limitado, por ter sido realizado com estudantes do ensino fundamental e médio de duas regiões do município do Embu, reflete a situação dos adolescentes que vivem em uma região periférica da região metropolitana de São Paulo frente à iniciação cada vez mais precoce do uso de drogas lícitas (álcool e tabaco) e sugere a importância do ambiente familiar na indução e facilitação deste uso.

Os resultados do estudo permitiram ações de prevenção e promoção à saúde, que estão sendo desenvolvidas pelo projeto de extensão universitária Corporalidade e Promoção da Saúde, junto às escolas de ensino fundamental e médio da região de Santo Eduardo e Santa Emília, município de Embu. De qualquer maneira, há necessidade de novos estudos em pontos distintos do território nacional, mais padronizados, a fim de fomentar a literatura nacional acerca do tema exposto.

\section{Agradecimentos}

Pelo apoio da Fapesp, por meio de bolsa de iniciação científica.

10. Ramirez RM, Andrade D. La familia y los factores de riesgo relacionados con el consumo de alcohol y tabaco en los niños y adolescentes. Rev Lat-Am Enfermagem 2005;13:813-8.

11. Schenker M, Minayo MC. Risk and protective factors and drug use among adolescence. Cienc Saude Coletiva 2005;10:707-17.

12. Zagury T. O adolescente por ele mesmo. Rio de Janeiro: Record; 1997.

13. Carlini EA, Galduróz JC, Noto AR, Nappo SA. I levantamento domiciliar sobre o uso de drogas psicotrópicas no Brasil: estudo envolvendo as 107 maiores cidades do país, 2001. São Paulo: Cebrid/EPM; 2002.

14. Carlini EA, Galduróz JC, Noto AR, Nappo AS, Fonseca AM, Carlini CM et al. II levantamento domiciliar sobre o uso de drogas psicotrópicas no Brasil: estudo envolvendo as 108 maiores cidades do país, 2005. Brasília: Senad; 2007.

15. Brasil - Ministério da Saúde. Vigilância de tabagismo em escolares: dados e fatos de 12 capitais brasileiras. v. 1. Rio de Janeiro: Inca; 2004

16. Callegari-Jacques SM. Bioestatística: princípios e aplicações. Porto Alegre: Artmed; 2003

17. Ventura RN, Oliveira EM, Silva EM, Silva NN, Puccini RF. Living conditions and infant mortality in the municipality of Embu, São Paulo, Brazil. Rev Paul Pediatr 2008;26:251-7.

18. Prefeitura da Estância Turística de Embu - Secretaria de Saúde. Programa de Integração Docente-Assistencial do Embu/Unifesp [cited 2008 Nov 16]. Available from http://www.unifesp.br/dped/embu/prgintdocemb. pdf 
19. Freud A. La adolescencia en cuanto perturbación del desarrollo. In: Caplan G, Lebovici S, editors. Psicologia social de la adolescencia. Buenos Aires: Paidós; 1974.

20. Urquieta JE, Hernández-Avila M, Hernández B. El consumo de tabaco y alcohol en jóvenes de zonas urbanas marginadas de México. Un análisis de decisiones relacionadas. Rev Salud Publica 2006;48:S30-40.

21. Kalina E. Psicoterapia de adolescentes. Rio de Janeiro: Francisco Alves; 1979.

22. Outeiral JO. Adolescer: estudos revisados sobre adolescência. 2nd ed. Rio de Janeiro: Revinter; 2003
23. Schenker M, Minayo MC. The importance of family in drug abuse treatment: a literature review. Cad Saude Publica 2004;20:649-59.

24. Nurco DN, Kinlock TW, O'Grady KE, Hanlon TE. Differential contributions of family and peer factors to the etiology of narcotic addiction. Drug Alcoho Depend 1998;51:229-37.

25. Horta RL, Horta BL, Pinheiro RT. Drugs: families that protect and that expose teenagers to risk. J Bras Psiquiatr 2006;55:268-72.

26. Figlie N, Fontes A, Moraes E, Payá R. Children of addicted parents with bio-psychosocial risk factors: do they need a special care? Rev Psiq Clin 2004;31:53-62. 